\title{
EPISODE FROM THE CREATE YOUR OWN SPACE ADVENTURE SERIES, IN WHICH A TYPICAL PREOCCUPATION WITH THE NATURE AND FUNCTIONS OF THE IMPERIAL COBALT MINES HAS LANDED YOU IN TIMES SQUARE, NEW YEAR'S EVE, 1948
}

\section{Bradley Paul}

You take the swizzle stick from you secret pocket. You're deft about things like this.

It has gathered quite some dust, which is unstable yet fragrant.

This reminds you of mishap $\mathrm{X}$ with regard to medium $\mathrm{Y}$,

which smacks of magnetized silver alloy. Your assistant, Gilette,

remarks on the dryness of Earth,

and at what megahertz Earth people ruin their sleep.

$\mathrm{He}$ is of loiterous complexion, with a cavalier gait. Sleep?

- Meanwhile, a kleenex is cut into squares before a window fan, and the night sky droops from star to star.

What can one appreciate from Times Square?

Gilette's martini glass is blown from mirrors,

a custom job, though surely Gilette

has not the lungs for that. Poplar trees were brought in for the season.

And you? There are popular tunes curbed inside your olive,

as there are logarithms back-burnered there, windowsful of afternoon

where something evil silled, fat 6's and unwrought 2's

crying "you bush-leagued Spanish pear, get it straight!"

So your cylinders jam, and your beakers, capsized,

pebble among the hydrogen. You think:

Gilette, he is of simple atomic weight, he is fine, he is slight,

he slurs into the elements near him. He is well-lionized

under such red light. And you? You are a broken-bottled drugstore,

you are the air-greened wires of Western Union,

you are the arctic circle at one o'clock last Thursday.

That playboy. You are the clergy of Aragon,

though you hate red. Velocity is pleasant. The kleenex stutter

across the pebbles: you remember your body. You want to hop a turnstile and turn left. You find your body scaled with miniature fire escapes. 
The sun is like a sunlamp. Don't you have a swizzle stick for situations like this?

Why are there turnstiles here in the arctic circle?

And what has Gilette been reading? 